\title{
Inclusión e interculturalidad para la cultura Sorda: caminos recorridos y desafíos pendientes
}

\author{
Inclusion and interculturality for the Deaf Culture: paths followed and pending challenges
}

\author{
Carolina Alejandra Becerra Sepúlveda
}

\begin{abstract}
RESUMEN
Esta investigación, realizada en Chile, se centra en la educación intercultural del Sordo. Busca conocer elementos comunes y divergentes que inciden en la relación dialógica entre cultura Sorda y oyente. El trabajo expone un análisis de los antecedentes teóricos que explican la realidad subalterna de esta cultura en el país, donde la mayoría del estudiantado asiste a escuela regular con Programas de Integración Escolar. Además profundiza en conceptos centrales de educación intercultural en un contexto de cultura Sorda. Ellos orientan una investigación empírica realizada en la región metropolitana de Santiago, cuyo objetivo consistió en identificar los elementos experienciales de la relación entre estudiantes Sordos y oyentes de una escuela con Programa de Integración Escolar que propenden al desarrollo de un proyecto intercultural. El estudio contempla un diseño etnometodológico y se interesa en la acción social entre la cultura Sorda y oyente. El trabajo de campo incorporó observaciones no participantes y focus groups. Sus resultados confirman la presencia de algunos elementos que propenden a la relación intercultural al interior de las aulas, los cuales se afectan por la modalidad comunicativa de ambos grupos. Ellos aparecen como consecuencia de la carencia de políticas públicas para garantizar los derechos lingüísticos del estudiantado Sordo.

Palabras clave: cultura, sordera, interculturalidad, educación, inclusión.
\end{abstract}

\begin{abstract}
This research, conducted in Chile, focuses on the intercultural education of the Deaf. It aims to recognize common and divergent elements that influence in the dialogic relationship between the hearing and Deaf culture. This work exposes an analysis of the theoretical background that explains the subordinate reality of this culture in the country, where most of the students attend a regular school with School Integration Programs. Besides, it delves into central concepts of intercultural education in a Deaf cultural context. They guide an empirical research conducted in the Santiago metropolitan region, whose objective was to identify the most outstanding elements of the relationship between Deaf and hearing students in a school with the School Integration Program that pursue the development of an intercultural project. This study takes into account an ethno methodological design and is interested in the social action between the Deaf and hearing culture. This field research integrates non participant observations and focus groups. Its results confirm the presence of some elements that favour the intercultural relation inside the classroom, which are affected by the communicative modality in both groups. They appear as a consequence of the lack of public policies to guarantee the linguistic rights of the Deaf students.
\end{abstract}

Keywords: culture, deafness, interculturality, education, inclusion. 


\section{INTRODUCCIÓN}

Desde su génesis, la educación de niños Sordos se ha visto anclada al paradigma clínico, transformando la escuela en un espacio terapéutico para el acceso a la lengua hablada. Esta postura encuentra oposición desde el paradigma sociocultural, que defiende el derecho del niño a aprender en su lengua materna dentro de la escuela, desarrollando competencias para su desempeño futuro en la sociedad. Situado en un enfoque de derechos, actualmente en Chile el paradigma sociocultural instala la discusión en torno a la facultad que le asiste al niño Sordo a acceder a una educación de calidad en Lengua de Señas Chilena (en adelante LSCh).

Hablar de educación para la cultura Sorda en Chile constituye un asunto controversial, que revela la paradoja entre el discurso y la praxis. Esto se visualiza en las precarias condiciones en que transcurre la experiencia educativa de los niños Sordos. Si bien el discurso desde las políticas públicas aboga por un enfoque de derechos hacia la diversidad Sorda, en la práctica existe una tendencia a la disminución de escuelas especiales, las cuales no se comprenden como espacios fundamentales para el desarrollo de una cultura Sorda que aprende en su lengua materna. En contraparte, encontramos un aumento sostenido de estudiantes Sordos que acceden al sistema de educación regular, en escuelas con Programas de Integración Escolar (en adelante PIE). Así, actualmente, del total de la población estudiantil Sorda matriculada en el sistema escolar, el 79\% de los estudiantes asiste a escuela regular con PIE, mientras que sólo el 21\% asiste a escuela especial (Ministerio de Educación, 2018).

Ante esta nueva realidad educativa de la población Sorda, la pugna entre el paradigma clínico y el sociocultural se traslada a la escuela regular, en la que, si bien se ha visto influenciada tempranamente por el enfoque clínico de la discapacidad, actualmente se evidencia una tendencia a reconocer el potencial de la comunidad Sorda para acceder a los aprendizajes. Pero lo anterior no supone el reconocimiento del carácter de esta comunidad como "cultura".

La escuela regular de hoy se enfrenta entonces no solo al desafío de modificar sus prácticas pedagógicas, sino también a la construcción de nuevas formas de concebir las relaciones sociales con la diversidad Sorda, instalando canales de reconocimiento (desde lo cultural) y comunicación efectivos, en un contexto de interculturalidad.

Carolina Alejandra Becerra Sepúlveda. Profesora-Investigadora de la Carrera Pedagogía en Educación Diferencial, Escuela de Educación de la Universidad Mayor, Chile. Es doctora en Educación y cuenta con especialidad en Interculturalidad por la Universidad de Santiago de Chile. Ha trabajado como profesora de aula en proyectos de integración escolar y como directora en escuelas especiales en Chile. Actualmente se desempeña como investigadora y académico en programas de pre y post grado para carreras de educación. Ha prestado asesoría para la institución de políticas públicas en Chile. Autora de múltiples investigaciones en el área. Expositora en congreso de Psicología Educacional, Interculturalidad, Inclusión y Educación especial. Correo electrónico: carolina.becerra@mayor.cl. ID: http://orcid.org/000-0002-8048-0707. 
La problemática ideológica de los paradigmas clínico y sociocultural instalados en la escuela regular constituye hoy un reto para la educación intercultural, que requiere redefinir su concepción acerca de la diversidad. Para el caso chileno esto es un imperativo, ya que en general existe una tendencia a asociar la idea de interculturalidad con pueblos originarios y migrantes, dejando a la comunidad Sorda en un estatus de minusvalía, donde sus integrantes sólo son beneficiarios de actividades de inclusión, sin un reconocimiento explícito de su lengua e identidad cultural.

Esta investigación sitúa su atención en una nueva concepción de la noción de sordera, que permita comprenderla como una cultura que vive procesos de inclusión e interculturalidad similares a los restantes casos de diversidad. Para ello, pretende observar el modo en que se producen experiencias inclusivas e interculturales en la escuela regular, en una búsqueda de acciones que orienten futuras estrategias de interacción simétrica en sus aulas.

\section{Cultura Sorda: un (corto) camino desde la diversidad}

El estudio de la comunidad Sorda como tópico de análisis cultural es nuevo, especialmente si se considera que el individuo Sordo ha sido considerado tradicionalmente como "discapacitado". Así, la noción de comunidad Sorda como cultura se enfrenta hoy a la clásica ideología "oralista", que defiende el aprendizaje del habla y niega el uso de las señas (Oviedo, 2007).

Diversos autores (Humphries, 1975; Lane, 1984; Fisher y Lane, 1993; Ladd, 2003; Bauman, 2004; Skliar, 2009; Hauser, O’Hearn, McKee, Steider y Thew, 2010; Holcomb, 2010; Ballenger, 2013; Cuevas, 2013) han participado en la génesis y/o reafirmación de esta idea, reconociendo, describiendo y analizando los debates en torno a los paradigmas clínico (oralista) y sociocultural (gestualista). Al respecto, la literatura señala la presencia de una profunda discusión respecto a las bases epistémicas de cada paradigma y sus estrategias para el abordaje del fenómeno educativo de la comunidad Sorda.

Lo señalado sitúa a la persona Sorda en una condición de exclusión ante situaciones que le afectan y competen. De esta manera, la comunidad Sorda ha pasado a constituirse en un grupo "Sordo subalterno" (Ladd, 2003), perdiendo su derecho a participar activamente en la toma de decisiones respecto a su realidad. Para Humphries (1975), la subalternidad tiene sus bases en la concepción de lo que denominó "audismo".

En términos generales, el audismo es un prejuicio relacionado con la condición física de audición y se manifiesta a menudo en actitudes paternalistas (Humphries, 1975; Lane, 1999; Bauman, 2004; Clark y June, 2013; Ballenger, 2013; Cuevas, 2013, Stapleton, 2016). Se trataría de una conducta manifiesta tanto en individuos oyentes como hipoacúsicos o Sordos educados bajo el paradigma clínico. Cuevas (2013) agrega 
que su arista más perniciosa es que "frecuentemente opera de manera inadvertida por vía de la benevolencia, que de modo inconsciente despolitiza y 'discapacita' a la comunidad de Sordos” (p. 704). El autor señala que el audismo tiene como consecuencia la aparición de privilegios y desventajas entre quienes lo experimentan, "lo que se traduce en efectos positivos de reconocimiento y emancipación, o negativos de invisibilización, negación y opresión de grupos humanos” (p. 699).

En oposición a los fundamentos ideológicos del paradigma clínico y del audismo, los miembros de las comunidades Sordas han levantado una definición de la sordera, como un término cultural. Así, un número cada vez mayor de personas sordas no se consideran a sí mismas como discapacitadas, sino como un grupo cultural distinto: con sus propias creencias, necesidades, opiniones, costumbres y lengua (World Federation of the Deaf, 2018).

En la actualidad, hablar de cultura Sorda es un tema polémico; la comunidad Sorda reclama una concepción social y denomina su condición de sordera con la letra "S" mayúscula. Algunos autores (Woodward, 1972; Ladd, 2003; Fernández, 2005) levantan una definición al respecto. Fernández (2005) señala que:

[...] se usa la convención de escribir "Sordo", con mayúscula, para denominar las personas cuya primera lengua es una lengua de signos y que tienen, con ella, especificidades culturales. Esto podría incluir personas que oyen, que por diferentes razones (la mayoría son familiares), han crecido usando una lengua de signos. Y la palabra "sordo", con minúscula, designa las personas que padecen deficiencia auditiva severa, sin discriminar el medio de comunicación que utilizan [p. 37].

En una búsqueda de las formas de verificación de esta cultura Sorda en Chile, De la Paz y Salamanca (2009) detallaron los siguientes elementos constitutivos: (1) lo simbólico (lengua de señas), (2) lo emotivo (desconfianza, frustración, incomunicación e inferioridad producto de la colonización lingüística), (3) conocimientos (transmisión de la cultura), (4) materiales (elementos propios de su cultura como la LSCh y sus manos).

La nueva concepción de la comunidad Sorda como cultura no solo consolida la identidad de este colectivo, sino además genera un sentimiento de orgullo que robustece la condición de pertenencia a una historia y lengua comunes. Esta nueva forma de aproximación a la condición Sorda ha sido definida por Ladd (2003) como "Sordedad".

Los fundamentos del movimiento Sordo subalterno y la idea de Sordedad (Ladd, 2003), como procesos experienciales propios de la cultura Sorda, cambian la tradicional perspectiva clínica de estudio de esta temática, centrándose ahora en la comunidad y las relaciones que establecen las personas Sordas con su entorno oyente. Estos nuevos intereses otorgan un campo de acción al enfoque inclusivo e intercultural, tal como se examinará a continuación. 


\section{Elementos fundamentales de la interculturalidad en un contexto Sordo/oyente}

Se define a la interculturalidad como "la presencia e interacción equitativa de diversas culturas y la posibilidad de generar expresiones culturales compartidas, a través del diálogo y del respeto mutuo" (UNESCO, 2006). Como concepto dinámico que atañe a las relaciones sociales, la interculturalidad es una tarea de reconocimiento a la diversidad fundamental de las sociedades contemporáneas y busca instalar experiencias de expresión cultural comunes, basadas en relaciones simétricas y respetuosas. En un contexto educativo, Treviño, Morawietz, Villalobos y Villalobos (2017) señalan que la educación intercultural "sería el resultado de cambios en las relaciones sociales -históricamente asimétricas- entre las poblaciones que habitan en un país, y daría cabida y representación a las tradiciones culturales de todos los estudiantes en un espacio que las valore a todas de igual manera" (p. 29). Besalú (2002) agrega que la interculturalidad es además una cuestión de carácter socio-político, ya que no se pretende educar en igualdad a toda la ciudadanía en un contexto heterogéneo, sino que se espera que constituya una oportunidad para aprender a convivir con toda la diversidad en un contexto inclusivo. Atendiendo a este carácter inclusivo es que la educación intercultural no se dirige sólo a las minorías étnicas o culturales, sino que es transversal a todos los integrantes de los grupos sociales, los cuales deben educarse para la convivencia y reconocimiento del otro.

Como espacio de reafirmación de la riqueza de la diferencia, el enfoque inclusivo e intercultural requiere de la articulación e instalación de prácticas que garanticen las relaciones dialógicas en un círculo virtuoso conformado, entre otros elementos, por: (a) identidad, (b) reconocimiento, (c) alteridad y (d) pluralismo.

En este orden de ideas, Becerra (2013) resignifica los requisitos esenciales que definen la interculturalidad, contextualizándolos en una perspectiva de cultura Sorda chilena, a través de los siguientes componentes que debieran estar presentes en la interacción dialógica e intercultural Sorda y oyente:

(1) Alteridad: corresponde a la toma de conciencia de las diferencias entre ambas culturas, logrando el reconocimiento y autoidentificación de parte de cada individuo, lo que permite el respeto de la diversidad intergrupal. Esto se traduce finalmente en el reconocimiento del otro como integrante de una cultura.

(2) Lengua: implica la valoración de la comunicación entre ambas culturas mediante el uso de una herramienta accesible para todos. Esto significa respetar la LSCh, abandonando toda pretensión de subordinarla a una lengua hegemónica hablada. Requiere postular la necesidad de aprendizaje de la LSCh por parte de la cultura oyente; mientras la cultura Sorda desarrolla el lenguaje oral escrito. Este intercambio lingüístico permite, además del desarrollo de 
la comunicación, el establecimiento de experiencias comunes entre ambas culturas.

(3) Intercambio cultural: que potencia la generación de experiencias comunes entre ambos grupos y por tanto borra los límites entre "lo normal y lo anormal". Demanda la prescindencia de conceptos de "rehabilitación", "normalización”, "integración”, “audismo” y "discapacidad”.

(4) Reciprocidad de experiencias: el beneficio lingüístico mutuo es evidente; es el que permite el intercambio de conocimientos y cosmovisiones propias de cada cultura, favoreciendo el desarrollo de un imaginario social que permita derribar los mitos instaurados por el paradigma clínico de la discapacidad. Esta reciprocidad tiene su origen en el pluralismo, como requisito y punto de partida de la valoración cultural.

Sustentada en el enfoque inclusivo, la educación intercultural supone una convivencia simétrica y dialógica, que incorpora diversas cosmovisiones y legitima sus voces, para la construcción de una nueva relación cultural. Si se insiste en su carácter inclusivo, entonces resulta imposible concebir la educación intercultural desde una mirada restringida a los pueblos originarios y migrantes (Dietz, 2017). En este sentido, la exclusión de otras diversidades implicaría una paradoja inexcusable tanto en términos epistémicos como prácticos. Y esto es así especialmente cuando se observan las similitudes entre los procesos de inclusión de la diversidad Sorda con las experiencias de pueblos originarios (Treviño et al., 2017), de la comunidad LGTBI (Rojas, Fernández, Astudillo, Stefoni, Salinas y Valdebenito, 2019), migrantes (Castillo, Santa-Cruz y Vega, 2018), entre otros.

La lógica del sustento teórico esbozado precedentemente no es posible de verificar en la práctica en Chile, donde la noción de interculturalidad sigue asociada a un factor contextual relacionado con la inmigración y los pueblos originarios. Esto coincide con lo señalado por Dietz (2017), quien acusa que el concepto de interculturalidad es frecuentemente utilizado para referir a la "diversidad 'provocada' por la migración, mientras que en otras la misma noción se aplica a para las interacciones entre pueblos indígenas y descendientes de colonizadores" (p. 192).

\section{Aproximaciones interculturales: la realidad de la cultura Sorda en Chile}

La educación inclusiva e intercultural instalada en las escuelas regulares demanda la provisión de estrategias para su atención. A nivel internacional, para el caso de la cultura Sorda, las realidades son diversas, destacando los avances en la experiencia mexicana y colombiana en educación superior y la española en educación escolar obligatoria. Todas ellas tienen un elemento común: la participación de un mediador intercultural, educador tradicional o co-educador Sordo, que media las relaciones 
sociales entre ambas culturas, favoreciendo el acceso a una comunicación efectiva.

En Chile la realidad es bastante distinta. Hasta el año 2017 los niños Sordos sólo tenían acceso a educarse en escuela especial hasta $6^{\circ}$ grado de educación básica. Esto significó que muchos de ellos tuvieran que integrarse en escuelas regulares una vez terminada su escolaridad especial. El resultado no siempre fue favorable: muchos se integraron a centros educativos que no cuentan con intérprete, lo cual es una barrera que dificulta su progreso en el currículum. Por otro lado, estos centros tampoco conocen la LSCh, ni reconocen a la comunidad Sorda como cultura.

Frente a esto se plantea la necesidad de fortalecer la formación del estudiante Sordo en escuela especial todo el tiempo que sea posible, educando a estos niños "para vivir en dos mundos" (Ladd, 2003) una vez egresados de la escuela especial. Para Ladd (2003) la educación del niño Sordo en escuela especial es un asunto realmente importante y reconoce el rol fundamental del educador Sordo como el actor esencial para la transmisión de actitudes asociadas a la Sordedad. Por ello destaca la importancia de contar con estrategias apropiadas dentro de la escuela especial para el progreso del alumno Sordo.

Atendiendo a esto, las investigaciones en Chile han centrado su atención en la observación de prácticas exitosas de la escuela especial para el desarrollo de la lectura y escritura (Morales, 2018, 2019; Herrera y De la Paz; 2019), el acceso al bilingüismo (Lissi, Svartholm y González, 2012; Herrera y De la Paz, 2017) y el rol del educador Sordo en contextos interculturales bilingües dentro de la escuela especial (De la Paz, González y Otárola, 2016).

Los avances en este ámbito contribuyen a la provisión de antecedentes para instalación de mejoras al interior de la escuela especial, aportando al aprendizaje del alumnado Sordo. Además permiten consolidar en la escuela especial una dinámica de interculturalidad crítica (Walsh, 2009), fundada en la Sordedad y el reconocimiento de las auténticas necesidades y cosmovisiones de la cultura Sorda.

A pesar de lo anterior, la realidad en Chile sigue una dirección contraria: existe un alto número de estudiantes Sordos integrados en escuelas con PIE, los cuales no se benefician directamente de la experiencia de escuela especial. Asimismo, la experiencia chilena demuestra que muchos niños egresados de escuela especial en $6^{\circ}$ grado de educación básica se ven forzados a integrarse en escuelas regulares que no manejan LSCh, lo que supone un golpe importante a su desarrollo y procesos educativos. Estas realidades son las que motivan el desarrollo de este estudio, que se pregunta acerca de la existencia de experiencias efectivas de inclusión e interculturalidad en escuelas regulares y se plantea la interrogante respecto al modo en que se desarrollan las relaciones sociales al interior de las aulas. Específicamente interesa a este estudio reconocer factores que contribuyen o interfieren en la instalación de experiencias 
interculturales al interior de las aulas de escuelas regulares, describiendo aspectos que deben considerarse para apoyar la inclusión y el derecho a una educación intercultural para la cultura Sorda. En este punto es importante señalar que la búsqueda de estos caminos no pretende dejar fuera la relevancia de la identidad de la cultura Sorda, la LSCh y la noción de Sordedad. El estudio fundamenta su abordaje en la perspectiva sociocultural y el enfoque de derechos de la cultura Sorda, reconociendo la identidad de este colectivo como condición para la instalación de relaciones interculturales con la comunidad educativa oyente.

\section{Método}

Esta investigación contempla un diseño de tipo etnometodológico. El estudio se interesa en la acción social, intersubjetividad y comunicación entre cultura Sorda y oyente. Todos estos tópicos pragmáticos son de interés de la etnometodología. Acuñado por Garfinkel (1967), el término “etnometodología” se refiere al estudio de cómo las prácticas o métodos son usados por los miembros de un grupo en circunstancias concretas, lo que permite una revaloración fundamental y un entendimiento detallado de la naturaleza de ese orden.

A través del diseño etnometodológico, lo que el estudio busca es conocer la naturaleza de las prácticas de los miembros de una relación intercultural, que se recrea constantemente en la cotidianeidad de la relación social.

\section{Objetivos}

\section{Objetivo general}

Analizar críticamente el papel que juegan los componentes interculturales comunes y divergentes presentes en la interacción entre la cultura Sorda y oyente, de una escuela de integración de la región Metropolitana (Chile), en tanto facilitadores u obstaculizadores en la construcción de espacios de diálogo intercultural, legitimación de la diversidad y potenciación de una relación culturalmente simétrica.

\section{Objetivos específicos:}

1. Identificar los elementos experienciales de la relación entre estudiantes Sordos y oyentes de una escuela con Programa de Integración Escolar de la región Metropolitana que propenden al desarrollo de un proyecto intercultural.

2. Identificar componentes de la educación intercultural que se potencian o inhiben en la interacción dialógica entre Sordos y oyentes.

3. Caracterizar las representaciones sociales acerca de la cultura Sorda y oyente en las experiencias comunes de comunicación y relatos de vida intercultural. 


\section{Población y muestra}

Los criterios de selección del establecimiento estuvieron basados en su trayectoria en inclusión de estudiantes Sordos usuarios de LSCh en sus aulas.

Para ello se buscaron establecimientos que cumplieran las siguientes condiciones:

(1) Contar con estudiantes Sordos usuarios de LSCh integrados en sus aulas.

(2) Poseer una trayectoria de al menos cinco años con Programa de Integración Escolar para estudiantes Sordos.

Asimismo, los estudiantes Sordos integrados debían cumplir con las siguientes características:

(1) Provenir de escuelas especiales adscritas al paradigma sociocultural de educación (LSCh).

(2) Presentar sordera congénita.

(3) Ser usuarios nativos de Lengua de Señas Chilena.

De esta manera se buscó asegurar que los participantes Sordos del estudio dieran cuenta de elementos característicos de la noción de Sordedad (Ladd, 2003).

En cuanto al establecimiento, el requisito de cinco años de experiencia en Programa de Integración Escolar buscó garantizar prácticas de interacción sostenidas en el tiempo, que tiendan a la instalación de prácticas interculturales.

Definidos los criterios de participación, se tomó contacto con la Unidad de Educación Especial del Ministerio de Educación de Chile, por ser esta la institución del Estado responsable de velar por la implementación de los PIE. A ella se le solicitaron antecedentes de experiencias de integración escolar favorables. A partir de la información provista se seleccionaron establecimientos que cumplieran con los requisitos del estudio, solicitando su participación. De un total de tres establecimientos que cumplían con los requisitos para la investigación, sólo uno accedió a participar. Esta institución corresponde a un Liceo Politécnico de la comuna de Santiago, que otorga enseñanza media técnico-profesional y atiende a estudiantes del género femenino.

Se tomó contacto con el equipo directivo, profesores, alumnos y apoderados, a quienes además de presentar los antecedentes del estudio y de la investigadora, se les solicitó consentimiento informado.

Previo al trabajo de campo se procedió a desarrollar un análisis documental de la información institucional, levantando un perfil del Liceo en relación a la educación de estudiantes Sordas. A partir de lo anterior, se puede afirmar que el Liceo cuenta con un Proyecto de Integración Escolar que atiende a estudiantes con déficit auditivo y motor. La institución imparte menciones de capacitación en administración, atención de enfermería, atención de párvulos y servicios de turismo. Su proyecto educativo institucional contempla, entre otros aspectos, erradicar la discriminación y propender a la atención y apertura a la diversidad de necesidades de sus estudiantes. Y es a partir 
de este que surge el Proyecto de Integración Escolar, que a la fecha de visita cumplió 13 años de implementación.

\section{Procedimiento de recogida y análisis de datos}

Se realizó una observación no participante en dos cursos compuestos por 34 estudiantes oyentes y 4 alumnas Sordas cada uno, alcanzando un total de 68 estudiantes oyentes y 8 estudiantes Sordas. El trabajo de campo se materializó en cuatro visitas a cada aula (jornada completa, incluyendo recreos), distribuidas en dos semanas. En todas las ocasiones se realizó una recolección de información en material de videograbación.

Finalizada la etapa de observación no participante (dos semanas), se realizaron focus groups con administrativos, docentes oyentes y estudiantes (Sordas y oyentes) y otro sólo con estudiantes Sordas. La primera etapa contó con un total de 61 estudiantes oyentes, 8 estudiantes Sordas, 6 docentes oyentes y 2 administrativos. El focus group exclusivo de estudiantes Sordas contó con 8 participantes.

En una tercera etapa se realizaron entrevistas en profundidad a cada una de las 8 alumnas Sordas integradas en los cursos observados (II y III Medio), con el fin de conocer su impresión acerca de las experiencias de interacción con la comunidad educativa oyente. En estas entrevistas se utilizó la modalidad comunicativa de LSCh, que la investigadora conoce y maneja de manera funcional.

Todas las técnicas de recopilación de datos buscaron información relativa a: (1) formas de interacción comunicativa entre alumnas Sordas y oyentes (elementos comunicativos comunes y divergentes); (2) objetivos que el Liceo pretende lograr con las alumnas integradas; (3) fines educativos que persiguen los docentes en sus alumnas integradas; (4) opinión de estudiantes oyentes respecto a experiencias de integración escolar; (5) historias educativas de las alumnas Sordas integradas; (6) semejanzas y/o diferencias culturales entre ambos grupos en el contexto escolar.

A partir de la información recolectada y contemplando las estrategias de análisis de contenido (Porta y Silva, 2003) y de doble mapeo (Taub, 2001), la investigadora levantó categorías, que fueron consignadas en una tabla de consistencia. Para efectos de análisis, ellas se organizaron en torno a dos tópicos fundamentales y basados en la literatura revisada: (1) elementos de representaciones sociales y (2) nociones de educación intercultural. Las categorías que se incorporaron en cada tópico se enumeran a continuación:

(1) Elementos de representaciones sociales. Agrupa categorías de: (a) subalternidad, (b) audismo y (c) cultura Sorda.

(2) Nociones de educación intercultural. Agrupa categorías que propenden a un proyecto inclusivo e intercultural, según la propuesta de Becerra (2013): (a) alteridad, (b) lengua, (c) intercambio cultural y (d) reciprocidad de experiencias. 


\section{Resultados}

A continuación se presentan los resultados obtenidos para cada uno de los tópicos y categorías levantadas:

\section{Elementos de representaciones sociales}

En este tópico se identificaron dispositivos experienciales de la relación entre Sordos y oyentes en el proyecto del establecimiento, teniendo como base la bibliografía revisada en torno a la cultura Sorda.

\section{a) Subalternidad}

Se evidencia que la principal característica de relación dominante de la cultura oyente radica en la opresión lingüística y el futuro acceso a la educación superior. Esto se observa tanto dentro como fuera de la sala de clases.

Dentro de la sala de clases esta opresión se manifiesta en la disposición espacial, que no favorece la comunicación a través de la LSCh. Los pupitres se distribuyen en filas, con lo que resulta imposible el intercambio comunicativo y el acceso a la LSCh tanto por parte de estudiantes Sordas como oyentes. Por otro lado, si bien existe una disposición y alto interés de los profesores y compañeras oyentes por aprender la LSCh, esta no constituye una vía de acceso fluida para los aprendizajes. Las clases se desarrollan con modalidad comunicativa bimodal, ${ }^{1}$ que restringe la LSCh a la estructura de la lengua hablada. Frente a esta situación sería deseable contar con un intérprete en LSCh, pero el Liceo informa que no posee recursos económicos para su contratación.

Si bien la situación descrita da cuenta de una posición subalterna de la LSCh frente a la lengua hablada, invisibilizándola y generando opresión lingüística, las estudiantes Sordas reconocen el interés de sus profesores y pares oyentes por comunicarse con apoyo de LSCh. A juicio de las estudiantes Sordas, el problema más importante a este respecto no surge de la experiencia educativa del Liceo, sino de los estatutos gubernamentales. Estas estudiantes exponen una percepción de abandono por parte de las autoridades y entidades encargadas de desarrollar políticas públicas para el acceso y permanencia de la cultura Sorda en el sistema de educación superior utilizando la LSCh como vía de comunicación. Así, denuncian escasas oportunidades que el sistema ofrece para su ingreso a la educación superior:

...ingresar a la Universidad es "un sueño" que eventualmente podría materializarse [...] sin embargo "se sabe" que eso es imposible sin intérprete...

1 Corresponde al uso simultáneo de LSCh y habla. Esta modalidad comunicativa no respeta la estructura sintáctica de la LSCh, sino mantiene a la base la estructura oral. 
Esta preocupación es compartida por sus pares oyentes, quienes señalan su decepción, por cuanto este no sería un tema que a las autoridades les interese (“...si no existen intérpretes en la sala de clases, será aún menos posible encontrarlos en la Universidad").

\section{b) Audismo}

Las participantes oyentes del estudio desconocen este concepto. A pesar de una exhaustiva observación de la realidad del Liceo, durante el desarrollo de esta investigación el audismo no aparece como conducta manifiesta. Esto no descarta, en todo caso, la posibilidad de que pudiera estar presente en otros contextos. La experiencia de este estudio evidencia que las relaciones espontáneas, tanto dentro como fuera de la sala, parecieran generarse en un plano que tiende a ser simétrico. Al consultar a las estudiantes Sordas, ellas no reportan experiencias de audismo, aún cuando aparece un caso de discriminación por parte de una profesora en particular.

No obstante lo anterior, el audismo se hace presente desde la figura del Estado, como una indiferencia al enfoque de derechos lingüísticos. Y esta se percibe como amenaza tanto desde la comunidad Sorda como oyente. Llama particularmente la atención que las compañeras oyentes manifiestan frustración frente al colonialismo lingüístico que desconoce el estatus de la LSCh (“...es ridículo, como si hablar nos hiciera superiores, tenemos que hacer algo"). Por tal motivo reclaman el derecho del Sordo a contar con intérpretes en todo el sistema educativo, erigiendo un enfoque de derechos en su concepción de la diversidad Sorda. En adición exigen la inclusión de un intérprete en el sistema de educación superior; no como un acto de eufemismo, caridad o benevolencia del Estado, sino como un derecho ciudadano ("¡El país tiene que hacer algo!’). Dada la falta de garantías respecto a la presencia de intérprete, las estudiantes oyentes ofrecen oficiar en este rol, pero reconocen que aquello puede mermar sus posibilidades para prestar atención a la clase. Y por tal motivo insisten en el rol del Estado y el enfoque de derechos:

...podríamos hacer de intérprete de alguna compañera Sorda, pero el ideal siempre es contar con un intérprete, para concentrarnos en los contenidos de la clase [...] esto es trabajo del Ministerio de Educación, no es un favor, es su obligación...

\section{c) Cultura Sorda}

Esta categoría es observada durante recreos, cuando las alumnas Sordas se reúnen de manera privada a compartir sus impresiones del día, experiencias y formas de entender la cotidianeidad, mediante el uso de LSCh. Se consultó la posibilidad de que esto obedeciera a una percepción de discriminación por parte de las compañeras oyentes, sin embargo las estudiantes Sordas lo niegan y señalan percibir aquello sólo de parte de una profesora. 
Los recreos son espacios privilegiados que las estudiantes Sordas valoran de manera especial. Ellas agradecen el lugar que les provee el Liceo (una sala del centro de alumnas), que se transforma en un espacio de enriquecimiento de los elementos constitutivos de la cultura Sorda descritos por De la Paz y Salamanca (2009). Se trata de espacios de cohesión, donde se fortalece lo simbólico (LSCh), los conocimientos (experiencias cotidianas relacionadas a la comunicación y necesidad de intérprete) y los materiales (uso del espacio para comunicarse con sus pares oyentes), todo ello sustentado en una noción de Sordedad, como experiencia exclusiva que les ofrece su condición.

Llama la atención el rol que toma lo emotivo (De la Paz y Salamanca, 2009). Los sentimientos de desconfianza, frustración, incomunicación e inferioridad descritos por los autores no sólo surgen desde la comunidad Sorda, sino que también aparecen en relatos desde la comunidad oyente, generando experiencias que tienden a lo intercultural. Las preocupaciones de las estudiantes Sordas en torno a su futuro laboral (“¿Quién va a querer contratar a una enfermera Sorda?”) y la falta de intérpretes en universidades y en el Liceo también son compartidas por las compañeras oyentes, que demandan los derechos comunicativos de la cultura Sorda:

...ellas pueden estudiar en la Universidad, pero el intérprete es un derecho, no un favor...

...además, nosotras también lo necesitamos...

Es probable que este constituyente emotivo, propio de la comunidad Sorda, esté abriendo espacios de alteridad en la comunidad oyente, que en este estudio en particular denuncia la opresión lingüística e interpela a la sociedad a un enfoque de derechos comunicativos:

...la sociedad también deberá aprender Lengua de Señas para que la mayoría pueda comunicarse con ellas y así la comunicación por este medio sea algo "natural"...

\section{Nociones de educación intercultural}

Como segundo tópico de organización se buscaron elementos constitutivos de la relación entre cultura Sorda y oyente que evidenciaran componentes que se potencian o inhiben en la relación dialógica intercultural. Para ello se consideró la caracterización propuesta por Becerra (2013).

\section{a) Alteridad}

Se evidencia la existencia de la noción de diversidad entre los grupos estudiados (Sordos y oyentes), sin embargo, ella no se asocia a un reconocimiento por parte de la comunidad oyente respecto a la existencia de una cultura Sorda. Las diferencias por parte de la comunidad oyente se caracterizan a partir del plano lingüístico, por la 
materialidad de la LSCh, y se acentúan dentro del aula, donde se ponen en evidencia las necesidades de comunicación de ambos grupos.

...en realidad, sólo tenemos idiomas diferentes... y nada más...

...ellas son iguales a nosotras...

\section{b) Lengua}

Los espacios de interacción observados durante la investigación valoran la comunicación para la optimización de los aprendizajes. Esto se evidencia no sólo en el interés de las estudiantes Sordas por realizar emisiones orales y gestos corporales espontáneos cuando estimen necesario, sino además en un claro esfuerzo de las alumnas oyentes por llevar sus gestos corporales espontáneos al ámbito de la LSCh y aprender esta modalidad de comunicación. ${ }^{2}$ Si bien los esfuerzos de las estudiantes oyentes no resuelven a completitud las barreras comunicativas y no se traducen en experiencias de bilingüismo, se trata de prácticas poco comunes en Chile. Esto se visualiza por cuanto las estudiantes oyentes incluso reclaman cursos gratuitos de LSCh (“...no todas tenemos plata para pagar un curso y también queremos aprender").

El valor de la comunicación es uno de los aspectos más interesantes evidenciados en este estudio. Además del reconocimiento de la LSCh como un "idioma” y la denuncia de la opresión lingǘstica por parte de las compañeras oyentes, se aprecia una cierta disposición a abandonar la supremacía oral, y una instalación espontánea de la comunicación signada, incluso entre personas oyentes (figura 1), aunque esto ocurra sólo en espacios informales dentro del aula y en recreos.

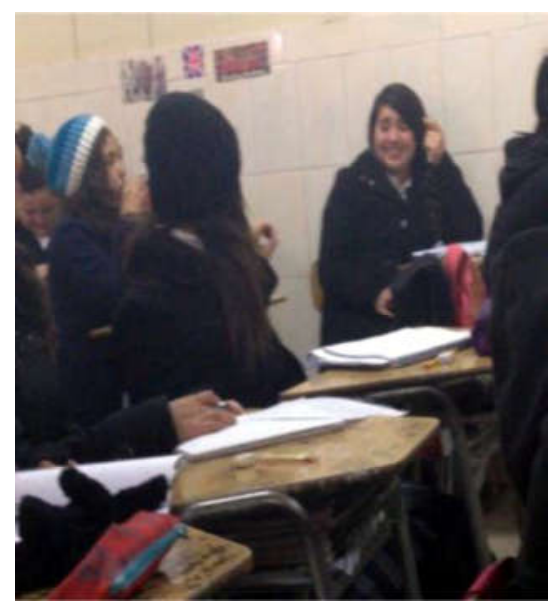

Figura 1. Comunicación en espacios informales. Compañeras oyentes se comunican mediante LSCh.

2 Un alto número de estudiantes declara tomar cursos certificados de LSCh. 
Estas situaciones producen diálogos sencillos en LSCh, cargados de la emotividad propia de esta lengua, en los que las estudiantes Sordas se evidencian empoderadas gracias a su código de comunicación. Esto genera espacios de distensión que valoran las manos como un órgano articulatorio. Y para tales efectos, se puede hacer callar a una persona Sorda tomando y golpeando sus manos, del mismo modo en que se cubren los labios del hablante cuando se le quiere silenciar (figura 2).

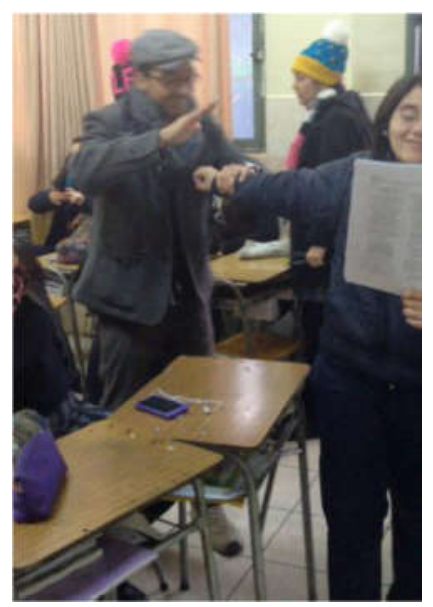

Figura 2. Las manos son órganos articulatorios.

Profesor hace callar a su estudiante para que "no hable leseras".

En ese acto golpea suavemente la mano de su estudiante Sorda.

El uso espontáneo de la LSCh se instala en la sala de clases durante breves momentos, creando espacios que propenden a la interacción intercultural. En ellos, Sordos y oyentes comparten experiencias sin recurrir a la oralidad (figura 3).

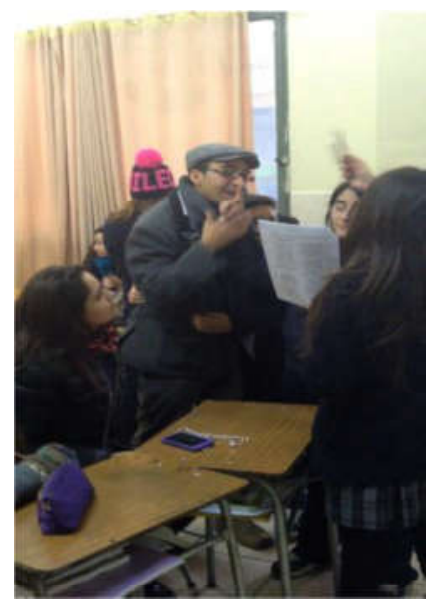

Figura 3. Conversación espontánea.

Profesor retroalimenta trabajo de su estudiante Sorda mientras sus compañeras prestan atención a la conversación en Lengua de Señas. 
A pesar de lo anterior, el valor comunicativo de la LSCh se interrumpe cuando se trata del desarrollo de la clase. El profesor retoma la modalidad comunicativa bimodal (figura 4) y la clase transcurre de manera simultánea en Lengua de Señas y hablada, mientras las compañeras interactúan entre sí, utilizando LSCh (figura 5) o gestos espontáneos (según el nivel de dominio de cada una).

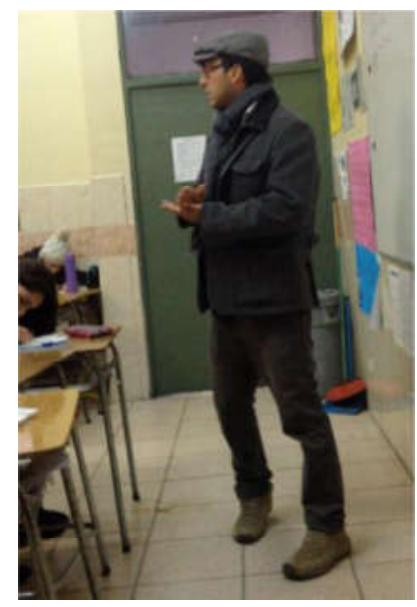

Figura 4. Modalidad comunicativa en aula.

Profesor habla y realiza la seña "hasta" simúltaneamente.

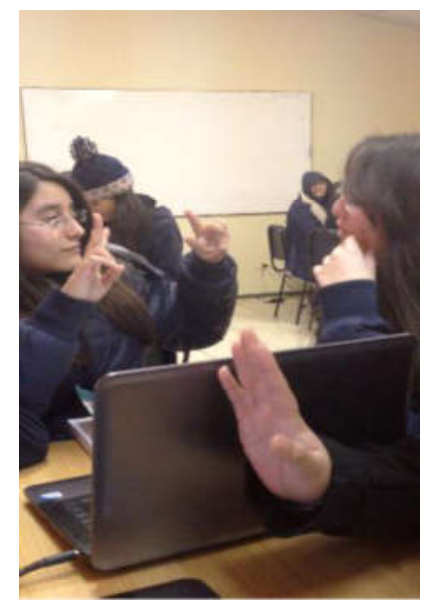

Figura 5. Conversación espontánea entre compañeras.

Estudiante Sorda explica a su

compañera oyente la lectura desarrollada.

\section{c) Intercambio cultural}

El reconocimiento e instalación de la LSCh en espacios informales de aprendizaje abre paso al intercambio entre ambas culturas, donde los valores y costumbres comunes se transforman en prácticas naturales (por ejemplo, apoyo mutuo en contenidos catalo- 
gados como "difíciles"). Este intercambio es apreciado y resulta muy beneficioso para ambos grupos en términos de aproximación a espacios interculturales de aprendizaje (figura 6), los que giran en torno a los contenidos curriculares en desarrollo.

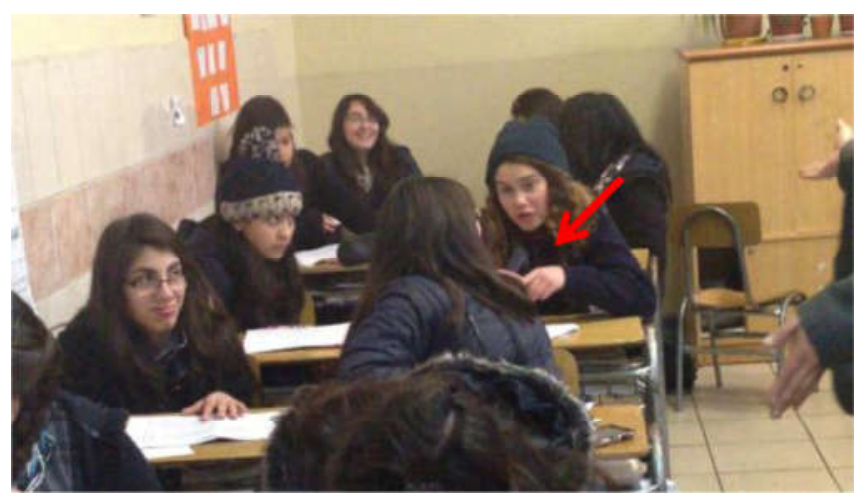

Figura 6. Experiencias de aprendizaje.

Ante los reclamos de una estudiante Sorda sobre la dificultad de los contenidos tratados, su compañera oyente reconoce que tampoco entiende bien, y que a pesar de que el profesor habla "dos idiomas", para ella también es difícil.

Nótese en la figura a una estudiante oyente signando "igual", para indicar "me cuesta igual que a ti".

A partir del intercambio cotidiano, los grupos propenden a la generación de experiencias comunes de aprendizaje, en las que destaca no sólo el apoyo que brindan las compañeras oyentes a sus pares Sordas sino también la ayuda académica (explicaciones) de alumnas Sordas a oyentes.

La situación descrita se ratifica en el focus group, donde las estudiantes oyentes señalan:

...me equivoqué al principio, pensé que a ellas les costaba aprender... ahora yo le pregunto a ella... y es bueno, me explica bien...

\section{d) Reciprocidad de experiencias}

De acuerdo a lo planteado por Becerra (2013), la reciprocidad de experiencias se asocia a un beneficio lingüístico mutuo, que permite el intercambio de conocimientos y elementos culturales propios de cada grupo, propiciando el desarrollo de un imaginario social. Si bien esta situación aparece de manera incipiente, se manifiesta en la comunicación espontánea dentro del aula, favoreciendo los aprendizajes para ambos grupos.

Como resultado se produce una valoración de las capacidades intelectuales de las estudiantes Sordas y reaparece en las compañeras oyentes la inquietud y crítica profunda ante la falta de políticas públicas que garanticen los derechos lingüísticos 
de la población Sorda, tanto en el Liceo como en la educación superior (“...esto es injusto").

Mientras que los profesores acusan falencias del sistema de educación superior, el cual, frente a estudiantes Sordas de excelencia, no cuenta con intérpretes para garantizar su derecho a la educación:

...la sociedad no está preparada para recibirlas $[\ldots]$

...la falta de lengua de señas es la principal barrera para el progreso de las alumnas en la sala de clases...

\section{Discusion Y CONCLUSIONES}

La educación intercultural es un tema que despierta reciente interés en las instituciones educativas, gatillada especialmente por fenómenos migratorios y demandas de grupos vulnerables asociados a género, pueblos originarios, comunidades étnico-religiosas, entre otros. En Chile esta experiencia se vincula con frecuencia a la multiculturalidad evidente en los grupos sociales, y se ha visto influenciada por políticas educativas para la educación intercultural en comunidades con estudiantes migrantes, minorías sexuales y pueblos originarios (mayoritariamente mapuche).

Si bien en este punto es importante reconocer sus alcances, es menester señalar la visión restringida de este concepto en Chile, donde en general la atención intercultural a la diversidad no se aplica a personas con discapacidad ni al colectivo Sordo en particular. La tendencia es a asociar la educación de este colectivo a una perspectiva clínica de la discapacidad, que en la práctica se traduce en políticas de integración escolar, aún cuando se refiere a ella como "inclusión”.

A pesar de que en Chile existen normativas para la inclusión de la discapacidad, estas no han sido suficientes para favorecer el avance curricular de los estudiantes Sordos en un contexto educativo intercultural. Así encontramos al 79\% del total de estudiantes matriculados asistiendo a centros de educación regular con PIE, sin que ello necesariamente sea respaldado con el apoyo de intérprete y profesor especialista en audición y lenguaje. A lo anterior se suma la tensión entre los paradigmas clínico y sociocutural instalados en la escuela regular, lo que ofrece un panorama poco alentador, caracterizado principalmente por un proceso de integración escolar, en circunstancias en que se aspira a la inclusión.

Considerando que la interacción entre comunidades Sorda y oyente en el sistema de educación regular es una realidad ineludible, este estudio analiza el papel que juegan los componentes interculturales en la interacción entre cultura Sorda y oyente en tanto obstaculizadores o facilitadores del diálogo simétrico. Para ello observó la presencia de factores asociados a la interculturalidad: (1) alteridad, (2) lengua, (3) intercambio cultural y (4) reciprocidad de experiencias, relacionándolos con las características 
propias de la cultura Sorda (De la Paz y Salamanca, 2009). Sus resultados no solo demuestran la presencia de estos cuatro factores, sino además revelan que ellos son elementos experienciales que efectivamente propenden al desarrollo de un proyecto intercultural (aun cuando resta un largo camino por recorrer), pero que lamentablemente son dependientes de la experiencia y disponibilidad del centro educativo.

Los resultados evidencian que los procesos de inclusión de la cultura Sorda a la educación regular son un asunto de muy lento progreso, que se afecta por las históricas discusiones entre los paradigmas clínico y sociocultural. Ellas se revelan en la instalación del oralismo y la opresión lingüística dentro de las políticas públicas y el sistema escolar, que no reconocen a la comunidad Sorda como cultura ni operativizan la instalación de la LSCh como lengua materna de los estudiantes Sordos. En efecto, este estudio evidencia el modo en que el oralismo permea las prácticas pedagógicas para la inclusión de estudiantes Sordos, las cuales se basan en la modalidad comunicativa bimodal, con lo que se obstaculiza la reivindicación de los derechos lingüísticos de este colectivo. Lo descrito se agudiza por la falta de políticas públicas que garanticen la presencia de un intérprete en aula regular, profundizando aún más la posición subalterna de la comunidad Sorda.

En contraparte, la mirada inclusiva del paradigma sociocultural al interior del Liceo estudiado se hace presente en el discurso de algunos docentes, que defienden la noción de inclusión y los derechos lingüísticos, realizando esfuerzos personales para aprender la LSCh. De esta manera, la nueva perspectiva instala en el debate la necesidad de políticas públicas para el reconocimiento del colectivo Sordo como cultura en un contexto inclusivo, promoviendo diálogos interculturales que fortalezcan la interacción simétrica con la comunidad oyente.

A partir de lo expuesto, conviene señalar que la superación de la perspectiva multicultural y su consecuentes intentos de integración escolar requerirán del fortalecimiento y reconocimiento de aquellas acciones que propenden al enfoque inclusivo e intercultural para las culturas Sorda y oyente. Del mismo modo, requerirá de un esfuerzo del Estado en la toma de conciencia respecto al modo en que sus políticas se encuentran obnubiladas por el enfoque clínico de la discapacidad y de la urgente necesidad de prestar atención a las demandas de la comunidad Sorda, presentes tanto en el sistema de educación especial como regular. Tales demandas se encuentran respaldadas además por las siguientes tres voces, que este estudio ha logrado recoger: (1) la comunidad educativa en general, (2) los compañeros de aula y (3) los docentes.

Desde la comunidad escolar se evidencia la urgencia de instalar mecanismos que permitan el reconocimiento del colectivo Sordo como cultura, incorporando la figura del co-docente Sordo para la mediación intercultural. Esto es fundamental, si se considera que la educación intercultural aboga por la preservación de los rasgos identitarios de las culturas y la defensa de las lenguas maternas. En un contexto 
Sordo, esto se traduce en la conservación de la Sordedad, para lo cual el co-docente Sordo es fundamental, desarrollando su quehacer del mismo modo que el educador tradicional (o mediador intercultural) labora en el centro educativo inclusivo para estudiantes migrantes o de pueblos originarios. Lo anterior no solo contribuye al diálogo intercultural y participación entre ambas comunidades, sino que trasciende más allá del aula, pudiendo aportar a la superación de experiencias subalternas de la cultura Sorda en la sociedad hegemónica.

Atendiendo a lo anterior, se estima que la llegada de adultos Sordos al sistema educativo contribuye al enriquecimiento de elementos esenciales que han sido descritos para la educación intercultural, valorando de manera especial la comunicación a través de la enseñanza de la LSCh. Este último punto se presenta como una oportunidad para apoyar los intentos de docentes y estudiantes por aprender esta lengua, superando la barrera económica y favoreciendo la presencia permanente de la LSCh en la escuela.

En relación a los compañeros de aula, la investigación evidencia el compromiso de la comunidad escolar oyente, que se apropia de aspectos emotivos descritos para la comunidad Sorda (De la Paz y Salamanca, 2009), adhiriendo a la defensa de la LSCh como modalidad comunicativa imprescindible en el sistema educativo. De esta manera se releva la figura del intérprete como un agente fundamental dentro del aula, del mismo modo en que ocurre con experiencias de niños migrantes que no manejan el idioma. Los hallazgos de este estudio reafirman la responsabilidad que le cabe al Estado en la instalación de mecanismos que permitan superar las barreras lingüísticas en la interacción intercultural.

Lo descrito anteriormente coincide con los discursos docentes, que reconocen el rol de la LSCh para el avance curricular del estudiantado Sordo. Esto lleva a respaldar las demandas de intérprete al interior de cada aula de clases, como una medida para garantizar el progreso en los aprendizajes.

Un aspecto que emerge como inquietud en este estudio se relaciona con una crítica profunda por parte de estudiantes y docentes oyentes a las políticas públicas en Chile, donde la falta de reconocimiento a los derechos lingüísticos de esta cultura no favorecen el progreso curricular ni permiten el acceso a la educación superior. La experiencia estudiada revela discursos comunes de reconocimiento por parte de la comunidad escolar a la identidad Sorda, la cual apoya las demandas de respeto a la lengua materna de las personas Sordas. Pero este reconocimiento no aparece de igual modo en el actuar del Estado, al cual se le demanda con fuerza iniciativas que debieran instalarse en la educación superior, velando por los derechos lingüísticos y educativos de un colectivo que ha sido tradicionalmente segregado.

Sustentada en el enfoque inclusivo, la educación intercultural para la cultura Sorda es un tema que aún no se encuentra suficientemente instalado en el ideario social nacional chileno. La falta de medidas para garantizar los derechos lingüísticos 
de esta población interfiere en las prácticas docentes para la inclusión de la cultura Sorda y limita su trayectoria escolar a las buenas intenciones de cada integrante de la comunidad educativa. Esta pobre mirada desde lo público no agota sin embargo el compromiso de la comunidad escolar, que construye estrategias creativas para fortalecer una relación dialógica que sólo se interfiere por la materialidad de la lengua. De esta manera se revela que las relaciones interculturales son una posibilidad manifiesta por el sistema educativo, pero que demanda un apoyo sistemático desde los órganos públicos y una toma de consciencia de su impacto en el progreso de la inclusión y educación intercultural de la población Sorda.

Este estudio demuestra que es posible generar un arraigo de los procesos de inclusión al interior de la comunidad educativa escolar, abriendo espacios a la interculturalidad (Dietz, 2011), el cual se soporta especialmente en los integrantes del sistema escolar y se obstaculiza por las políticas públicas. En otras palabras, este arraigo radica en la voluntad del sistema escolar y de cada escuela por llevar a cabo esta tarea, no existiendo garantías de replicabilidad debido a la escasa intervención del Estado en este ámbito. Incluso es necesario recordar que el estudio se realizó en un Liceo que contaba con 13 años de experiencia en integración de estudiantes Sordos, lo que le sitúa en una posición privilegiada respecto a otros centros educativos.

Los hallazgos de este estudio revelan un camino recorrido, donde la convivencia dialógica entre ambas culturas es una posibilidad cierta, aun cuando se base en esfuerzos conscientes de alteridad, reciprocidad y anhelos de intercambio intercultural. Esta posibilidad se debilita sin embargo por la materialidad de la LSCh y la postura audista de los órganos estatales, que no cuentan con políticas públicas para garantizar los derechos lingüísticos de esta población. Es probable que aquello incida en la persistencia de la subalternidad y la falta de reconocimiento del colectivo Sordo como cultura por parte de la sociedad oyente, transformándose en un desafío pendiente.

La observancia de factores asociados a la educación intercultural permite sostener que la explicitación de la diversidad Sorda en la noción de interculturalidad constituye una oportunidad para resignificar la noción de sordera, asociándola a un constructo cultural que favorece las relaciones dialógicas. Esto contribuye al debate ya planteado por la UNESCO (2019) en la búsqueda de nuevas miradas a la diversidad, ampliando el concepto de interculturalidad más allá de pueblos originarios y migrantes.

\section{REFERENCIAS}

Ballenger, S. (2013). Strategies to avoid audism in adult educational settings. Adult Learning, 24(3), 121-127.

Bauman, H. D. (2004). Audism: Exploring the metaphysics of oppression. Journal of Deaf Studies \& Education, 9(2), 239-246.
Becerra, C. (2013). Interculturalidad y ciencias de la educación. Boletín Cientifico Sapiens Research, 3(2), 2-7.

Besalú, X. (2002). Diversidad cultural y educación. Madrid: Síntesis.

Boatner, M. y Gates, J. (1975). A dictionary of American idioms. New York: Barron's Educational Series. 
Castillo, D., Santa-Cruz, E. y Vega, A. (2018). Estudiantes migrantes en escuelas públicas chilenas. Calidad en la educación, (40), 18-40.

Clark, R. y June, A. (2013). Audism: A theory and practice of audiocentric privilege. Humanity \& Society, 37(2), 101-130.

Cuevas, H. (2013). El gobierno de los Sordos: el dispositivo educacional. Revista de Ciencia Politica, 23(3), 693-713.

Conrad, R. (1979). From gesture to language in hearing and deaf children. Gallaudet University Press.

De la Paz, V., González, M. y Otárola, F.: (2016). Deaf educators: Linguistic models in an intercultural-bilingual educational context. En B. Gerner y L. Karnopp (eds.), Change and promise. Biligual Deaf education and Deaf culture in Latin America (pp. 82-102). Washington, DC: Gallaudet University Press.

De la Paz, V. y Salamanca, M. (2009). Elementos de la cultura Sorda: una base para el curriculum intercultural. REXE. Revista de Estudios y Experiencias en Educación, 8(15), 31-49.

Dietz, G. (2017). Interculturalidad: una aproximación antropológica. Perfiles Educativos, 39(156), 192-207.

Dietz, G. y de Mateos, L. (2011). Interculturalidad y educación intercultural en México. Un análisis de los discursos nacionales e internacionales en su impacto en los modelos educativos mexicanos. México: Secretaría de Educación Pública.

Fernández-Viader, M. (2005). El valor de la mirada. España: Universitat de Barcelona.

Fisher, R. y Lane, H. (1993). Looking back: A reader on the bistory of Deaf Communities and their Sign Languages. Hamburgo: Signum.

Furth, H. (1973). Pensamiento sin lenguaje: implicancias psicológicas de la sordera (R. Bellas y R. Martínez, trads.). Madrid.

Garfinkel, H. (1967). Studies in ethnomethodology. Englewood Cliffs, New Jersey. Unites States of America: PrenticeHall.

Hauser, C., O'Hearn, A., McKee, M., Steider, A. y Thew, D. (2010). Deaf epistemology: Deafhood and deafness. American Annals of the Deaf, 154(5), 486-492.

Herrera, V. y De la Paz, V. (2017). Lectores Sordos bilingües: un logro posible. RIL Editores.

Herrera, V. y De la Paz V. (2019). Prácticas pedagógicas y transformaciones sociales. Interculturalidad y bilingüismo en la educación de Sordos. Revista Latinoamericana de Educación Inclusiva, 13(1), 73-88.

Holcomb, T. K. (2010). Deaf epistemology: The deaf way of knowing. American Annals of the Deaf, 154(5), 471-478.

Humphries, T. (1975). Communicating across cultures (deaf=hearing) and language learning (tesis de doctorado inédita, Union Institute and University, Cincinnati, $\mathrm{OH}$ ).
Ladd, P. (2003). Comprendiendo la cultura Sorda. En busca de la Sordedad. Concepción: Consejo Nacional de la Cultura y las Artes.

Lane, H. (1984). When the Mind Hears. Nueva York: Random House.

Lane, H. (1999). The mask of benevolence: Disabling the Deaf community. Nueva York: Alfred A.

Lissi, M., Svartholm, K. y González, M. (2012). El enfoque bilingüe en la educación de Sordos: sus implicancias para la enseñanza y el aprendizaje de la lengua escrita. Estudios Pedagógicos, 38(2), 299-320.

Ministerio de Educación (2018). Informe de Gestión PIE Mineduc (inédito). Chile: Mineduc.

Morales, G. (2018). Diversidad Sorda: educación y sensibilidad intercultural en una escuela especial de Santiago de Chile. Psicogente, 21(4), 458-475.

Morales, G. (2019). Percepciones sobre la lengua de señas chilena en la educación de estudiantes Sordos: docente y codocente como sujetos históricos situados comunicativamente en el aula. Revista Educación, 43(2).

Oviedo, A. (2007) La cultura sorda. Notas para abordar un concepto emergente. Recuperado de: www.cultura-sorda.eu.

Porta, L. y Silva, M. (2003). La investigación cualitativa: el análisis de contenido en la investigación educativa. Mar del Plata.

Rojas, M., Fernández, M., Astudillo, P., Stefoni, C., Salinas, P. y Valdebenito, M. (2019). La inclusión de estudiantes LGTBI en las escuelas chilenas: entre invisibilización y reconocimiento social. Pensamiento Educativo. Revista de Investigación Educacional Latinoamericana, 56(1), 1-14.

Skliar, C. (2009). Educación, educar a cualquiera y cada uno. Sobre el estar-juntos en la educación. Santiago: UNESCO/ OREALC.

Stapleton, L. (2016). Audism and racism: The hidden curriculum impacting Black d/Deaf College Students in the Classroom. The Negro Educational Review, 67(1-4).

Taub, S. (2001). Language from the body. Cambridge: University Press.

Treviño, E., Morawietz, L., Villalobos, C. y Villalobos, E. (2017). Educación intercultural en Chile. Experiencias, pueblos y territorios. Ediciones Universidad Católica de Chile.

UNESCO (2006). Directrices sobre Educación Intercultural. Orientaciones. Santiago: UNESCO.

UNESCO (2019). Derecho a la educación e inclusión en América Latina y el Caribe. Ciclo de Webinars Informe GEM Regional 2020.

Walsh, C. (2009). Interculturalidad crítica y educación intercultural. Seminario Interculturalidad y Educación intercultural, La Paz. 
Woodward, J. C. (1972). Implications for sociolinguistic research among the deaf. Sign Language Studies, 1(1), $1-7$.
World Federation of the Deaf (2018). Declaración de la DMA en el debate general, $11^{\circ}$ reunion del COSP a la CDPD. Recuperado de: http://wfdeaf.org/news/resources/13-june-2018-wfdstatement-general-debate-11th-session-crpd/.

Cómo citar este artículo:

Becerra Sepúlveda, C. A. (2020). Inclusión e interculturalidad para la cultura sorda: caminos recorridos y desafíos pendientes. IE Revista de Investigación Educativa de la REDIECH, 11, e792. doi: https://doi.org/10.33010/ie_rie_rediech.v11i0.792. 